\title{
Some results on the distribution of additive arithmetic functions III
}

by

\author{
Q. Jogksm Bant (Calcutta, India)
}

0. Introduction. A real-valued arithmetic function $h$ is said to have a distribution if there exists a distribution function $G(x)$ on the real line such that the density of the set $\{m \geqslant 1: h(m)<x\}$ of positive integers existis and is equal to $G(x)$ at each continuity point $x$ of $G(x)$.

It is known that the distribution of a real-valued additive arithmetic function $f$, if it exists, is prore, that is, either discrete, continuous singular or absolutely continuous. It is also known that the distribution of a realvalued additive arithmetic function is discrete iff $\sum_{f(x) \neq 0} 1 / p<\infty$.

In 1939 P. Erdös [3] has shown that, if $f$ is a real additive arithmetic function given by

$$
f(p)=o\left(\frac{1}{p^{c}}\right)
$$

for all prime numbers $p$ and for some positive constant $c$, then the distribution of $f$ exists and is singular (i.e., either discrete or continuous singular). In this paper we show that if, for some $e>0$,

$$
\sum_{\substack{p \rightarrow N \\ p+Q}} \frac{\{f(p)\}^{2}}{p}=o\left(\frac{1}{N^{c}}\right) \text { as } N \rightarrow \infty
$$

whore $Q$ is a set of prime numbers such that

$$
\sum_{p \in Q} \frac{1}{p}<\infty
$$

then the distribution of $f(m)-f(m+1$ ) exists and is singular (i.e., either discrete or continuous singular). From this result we shall deduce that if $f$ satisfies (1) and $f$ has a distribution, then the distribution of $f$ is singular. In particulas, every bounded real-valued additive arithmetic function has a singular distribution. We shall obtain similar results for 
the distribution of values of $f(F(m))$, where $F(m)$ is an integral polynomial taking positive valtues for $m=1,2, \ldots$

Host of the proofs depend on the following observation. Let $f$ be a real-valued additive arithmetic function, having a distribution. The distribution of $f$ is singular (absolutely continuous) iff the distribution function corresponding to the characteristic function $g(t)$ given by

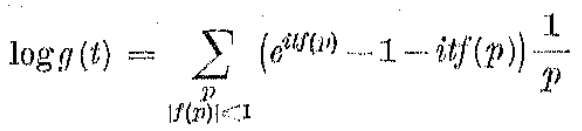

is singular (absolntely continnous).

\section{Notations and definitions.}

$p, q, p_{1}, p_{2}, \ldots$ always denote prime numbers.

$N, k, t, m, n$, ete., with or without suffixes always donoto positivo integers.

$\mathrm{P}\{\ldots\}$ denotes the probability of the erent in $\{\ldots\}$. For any random variable $X, L(X)$ denotes the distribution function corresponding to the random variable $x$.

$\omega(m)$ denotes the number of distinct juime divisors of $m$.

$c_{1}, c_{2}, \ldots$ denote constants.

\section{Results.}

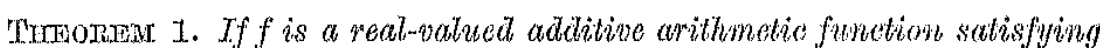
(1), then the distribution of $f(m)-f(m+1)$ exists and is singutor.

Tinesizun 2. If $f$ is a real-valued additine arithwetio function having an absolutely continnuous distribution, then, the distribution of $f(m)-f(m+1)$ is absotutely continuous.

Coroldary 1. Suppose $f$ is a real-valuet additive arithmetio function having a distribution. If $f$ satisfies condition (1), then the distribution of $f$ is singular.

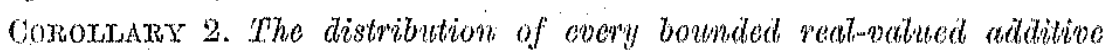
arithmetio function is singulas. In particular, wo additine anthmetio fundion can have a uniform distribution.

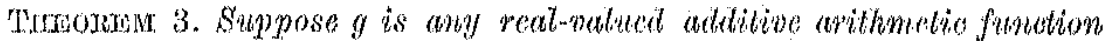
for whith there exists a constant $x$ such that

$$
\text { (2) } \quad|g(m)-g(m+1)|<K \quad \text { for } \quad w=1,2, \ldots
$$

Then the distribution of $g(m)-g(m+1)$ exists and is singuilar. fying

Irmown 4. Let $f$ be a reat-valued additive arithwetic funation satis-

$$
\liminf _{b \rightarrow 0}\left(1 / s^{2}|\log \varepsilon|\right) \sum_{\left|f_{p}\right|<\varepsilon} \frac{f_{y}^{2}}{p}>4
$$

where

$$
f_{y}=\left\{\begin{array}{lll}
f(p) & \text { if } & |f(p)|<1, \\
1 . & \text { if } & |f(p)| \geqslant 1 .
\end{array}\right.
$$

Then the distribution of $f$ is absolutely continuous.

Jet ilenote the set of all polynomials $Z^{\prime}$ with integral coefficients satisfying the following conditions:

(i) $m(m)>0$ tor $m=1,2, \ldots$

(ii) $t h$ is noti divisible by the square of any iredncible polynomial.

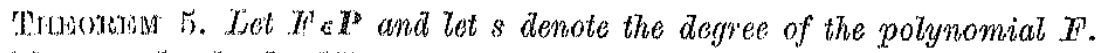
Let $f$ be a reditwalues additive arithmetio function satisfying

(3) $f\left(y^{b}\right) r(F, p) \rightarrow 0$ as $p \rightarrow \infty$ for $t=1, \ldots, s-1$, if $s \geqslant 2$,

$$
\sum_{\substack{p>N \\ x+(,)}} \frac{\{f(p)\}^{2} r(f, p)}{p}=0\left(\frac{1}{N^{c}}\right) \text { as } \quad N \rightarrow \infty
$$

where $4(T, t)$ denoles the number of incongruent sotutions of the congruence retation.

$$
h^{\prime}(m) \equiv 0(\bmod t)
$$

o is a yositive enstant and $Q$ is a set of primes such that

$$
\sum_{x \in Q}^{Y} \frac{r(F, p)}{p}<\infty .
$$

Then the distribution of $f\left(H^{\prime}(m)\right)$, if it exists, is singular.

Theornas 6. Under the conditions of Theorem $5, f(F(m))-f(F(m+1))$ has a singular distribution.

Theoram 7. With the same notation as above, suppose

$$
\operatorname{limint}_{s \rightarrow 0}\left(1 / s^{2}|\log \varepsilon|\right) \sum_{|f(p)|<s} \frac{(f(p))^{2} r(F, p)}{p}>4
$$

then the distribution of $f\left(F^{\prime}(m)\right)$ is absolutely continuous.

\section{Prelimimary results.}

Tiwma 1. If $\left\{x_{n}\right\}$ and $\left\{X_{n}\right\}$ are tiwo sequences of discrete and independent random nariables defined on the same probability space satisfying

$$
\sum_{n} \mathrm{P}\left\{X_{n} \neq Y_{n}\right\}<\infty
$$

then $\sum X_{n}^{r}$ oomverges almost overywhere and $L\left(\sum X_{n}\right)$ is absolutely continuous (singutan) iff $\sum_{n} X_{n}$ converges almost everywhere and $I\left(\sum_{n} Y_{n}\right)$ is absolutely continnums (singfultar).

The proof of this lemmat is woll-known. 
LEMMA 2. Let $f$ be a real-valued additive arithmetic funotion satisfying

$$
\sum_{p} \frac{\left\{f_{p}\right\}^{2}}{p}<\infty
$$

Let $\left\{X_{p}\right\}$ be a sequence of independent random variables with

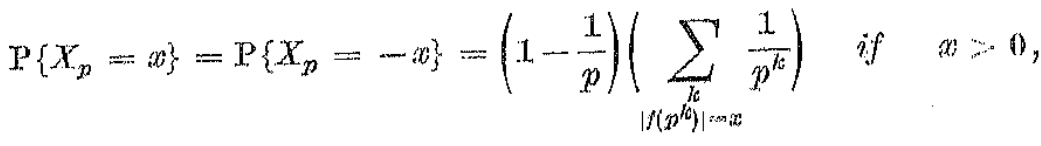

$$
\begin{aligned}
& \mathrm{P}\left\{X_{p}=0\right\}=1-\frac{2}{p}+2\left(1-\frac{1}{p}\right)\left(\sum_{\substack{p_{k} \\
f\left(p^{2}\right) \cos 0}} \frac{1}{p^{b}}\right) \text {. }
\end{aligned}
$$

Then $\sum_{n} X_{p}$ converges almost everywhere and the distribution of $f(m)-f(m+1)$ is $L\left(\sum_{p} X_{p}\right)$.

This result is contained in the proof of Proposition 1 [2].

Linura 3. If $F \in \mathbb{P}$, there exists a $p_{0}$ such that $p>p_{0}$ implies $r\left(f, p^{l}\right)$ $=r(F, p)$ for any $t \geqslant 1$. Also

$$
f(F, a \cdot b)=r(F, a) r(F, b) \quad \text { if } \quad(a, b)=1
$$

and

$$
r\left(t h, p^{t}\right) \leqslant x \text { for some integer } 7 \text { a depending only on } H^{4} \text {. }
$$

See [6].

LEMMA 4. For any positive integer $k \geqslant 2$ and if $x \geqslant 3$ we have

$$
\sum_{m \leqslant o} z^{o(m)}=\left(\varphi(k)+o\left(\frac{1}{\log x}\right)\right) \infty(\log x)^{k-1}
$$

where the constant induced by $O\left(\frac{1}{\log x}\right)$ depends only on $k$, and

$$
p(k)=\frac{1}{(k-1) !} \int\left(1-\frac{1}{p}\right)^{k}\left(1+\frac{k}{p-1}\right) .
$$

See Kubilins [б] ], p. 140 .

Lrearara 5. For any $n \geqslant 3$ and $k \geqslant 2$, we have

$$
\sum_{m \leqslant \leqslant} \frac{k^{\omega(m)}}{m}=\frac{\varphi(k)}{k}(\log n)^{k}+O(\log n)^{k-1}
$$

where the constant induced by $O(\log n)^{k-1} d e p e n d s$ only on $k$.
Proof. Partial summation gives

$$
\begin{aligned}
\sum_{m=1}^{n}\left(\frac{h^{\omega(m)}}{m}\right) & =\frac{1}{n} \sum_{m=1}^{n} k^{\omega(m)}+\int_{1}^{n} \frac{1}{x^{2}}\left(\sum_{m \leqslant x} k^{\omega(m)}\right) d x \\
& =O(\log n)^{k-1}+\int_{2}^{n} \frac{p(k)(\log x)^{k-1}+O(\log x)^{k-2}}{x} d x \\
& =O(\log n)^{k-1}+\frac{p(k)}{k}(\log n)^{k} .
\end{aligned}
$$

Tinw.M. 6. Sowppose $\left\{a_{p}\right\}$ is a sequence of real numbers satisfying

$$
\sum_{p>N} \frac{a_{m}^{2}}{p}=O\left(\frac{1}{N^{e}}\right) \quad \text { as } \quad N \rightarrow \infty \quad \text { for some } e \text {. }
$$

Irurther, suppose $g(t)$ is defined by

$$
g(t)=\exp \left\{\sum_{p}\left(e^{i t a_{p}}-1-i t a_{p}\right) \frac{1}{p}\right\} .
$$

Then $g(b)$ is a characteristic function of a distribution function and the distribution, function corresponding to $|g(t)|^{2 \gamma_{6}}$ is singular for any integer $f \geqslant 1$.

Proof. It is clear that $g(t)$ is a characteristic function. Fix an integer $\eta_{0} \geqslant 1$. Tet $\left\{X_{y 3}, X_{q}: p\right.$ and $q$ are primes greater than $\left.2 \%\right\}$ be a set of independent random variables satisfying for each $p>2 \%$

$$
\mathrm{P}\left\{X_{p}=t\right\}=\mathrm{P}\left\{Y_{p}=-t\right\}=\left(\frac{\hbar}{p}\right)^{t}\left(1-\frac{k}{p}\right) \text { for } t \geqslant 0 .
$$

Note that for any integer $t \geqslant 0$

$$
\mathrm{P}\left\{X_{p}+Y_{p}=-t\right\}=\mathrm{P}\left\{X_{p}+Y_{p}=t\right\}=\left(\frac{k}{p}\right)^{t}\left(1+O\left(\frac{1}{p^{2}}\right)\right) e^{-2 k / p} .
$$

In viow of $(6)$ and lumma $t$, it follows that $\sum_{p} a_{p p}\left(X_{p}+Y_{p}\right)$ converges almost overywhere and $L\left(\sum_{p} a_{p}\left(X_{p}+X_{p}\right)\right)$ is singular iff the distribution corresponding to $|g(t)|^{2 \%}$ is singular.

Without lows of generality wo ean assume $c<1$, in $(6)$. Let $N$ be a large intiger. Jut $m<N^{0 / 6}$ and

Consider tho sot

$$
m=p_{1 m}^{k_{m 1}} \ldots p_{t_{m}^{m}}^{k_{m} l_{m}}, \quad k_{m i} \geqslant 1 .
$$

$$
D_{m N}=\left\{\sum_{i m i}^{i_{m}} \varepsilon_{i} k_{m i l} a_{s_{i m}}: \varepsilon_{i}=+1 \text { or }-1, i=1, \ldots, t_{m\}}\right\} \text {. }
$$

Put $D_{N^{*}}=\bigcup_{m<N^{o / 6}} D_{m N}$. 
Since there are $2^{\omega(n)}$ sequences $\left(\varepsilon_{1}, \ldots, \varepsilon_{l, m}\right)$ of +1 and -1 , and

$$
\begin{aligned}
& \mathrm{P}\left\{X_{p_{i m}}+X_{p_{i m}}=\varepsilon_{i} k_{m i},\right. \\
&\left.i=1, \ldots, t_{m} \text { and } X_{p}+Y_{p}=0 \text { if } p \leqslant N \text { and }(p, m)=1\right\}
\end{aligned}
$$

is the same for any sequence $\left(\varepsilon_{1}, \ldots, \varepsilon_{t_{m}}\right)$ of +1 and -.1 , we have

$$
\begin{aligned}
& P\left\{\sum_{p \in B_{N}} a_{p}\left(X_{p}+Y_{p}\right) \in D_{N}\right\}
\end{aligned}
$$

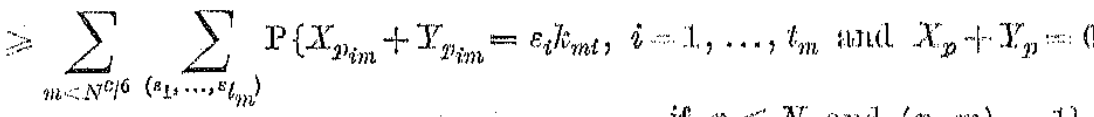

$$
\begin{aligned}
& \geqslant \sum_{m<N^{c / 0}} 2^{\omega(n)} \frac{z^{\omega(n)}}{m} \prod_{p \leqslant N}\left(1+o\left(\frac{1}{p^{2}}\right)\right) \exp \left\{-2 k \sum_{j \xi \xi N} \frac{1}{p}\right\} \text {. }
\end{aligned}
$$

Since $\sum_{p \in N} 1 / p=\log \log N+O(1)$, by Lemma 5 it follows that

$$
\mathrm{P}\left\{\sum_{p \leqslant N} a_{1,}\left(X_{p}+X_{p}\right) \in D_{N}\right\} \geq a>0
$$

for some constant a and for all large $N$.

Put $h=[0 / 3]+1$. For all sufficientily large $N$, wo have,

$$
\begin{aligned}
& P\left\{\left|\sum_{n>N} a_{p}\left(X_{p}+Y_{n}\right)\right|>N^{-c / 3}\right\} \\
& \leqslant P\left\{\sum_{p>N}\left|a_{p}\left(X_{p}+X_{p}\right)\right|>N^{-c / 3} \text { and for all } p>N,\left|X_{p}+X_{p}\right|<h+2\right\}+ \\
& -10\left(\sum_{p>N}^{1} \frac{1}{p^{h-1-2}-2}\right) \\
& =O\left(\sum_{p>N} \sum_{t=1}^{h+1} \frac{t^{2} a_{p}^{2} h_{i}^{l}}{p^{t}}\right) N^{2 e / 3}+o\left(\sum_{x>>N} \frac{1}{p^{2}}\right) N^{-h} \\
& =\theta\left(N^{-c / 3}\right)<b N^{-\sigma / 3} \text { for some } b>0 \text {. }
\end{aligned}
$$

So

$$
\mathrm{P}\left\{\sum_{p} a_{p}\left(X_{p}+Y_{p}\right) \epsilon G_{N}\right\} \geqslant a-b N^{-c / 3}>a / 2>0 .
$$

for all sufficiently large $N$, where $G_{N}=\bigcup_{d \in D_{N}}\left[a-N^{-6 / 3}, d+N^{-c / 3}\right]$. By temma 4 , the Lebesgue measure of the set $a_{N}^{N}$ tends to zaro as $N \rightarrow \rightarrow \infty$. Fonee $L\left(\sum_{p} a_{p}\left(X_{p}+Y_{p}\right)\right)$ is singrlar. This completes the proof of the lemma.

\section{Proofs of the theorems.}

Proof of Theorem 1. By (1) and Lemma 2, we can find a sequence $\left\{X_{p}\right\}$ of indepondent random variables satisfying (5) and the distribution of $f(m)-f(m+1)$ is $I\left(\sum_{p} X_{p}\right)$. It is not hard to find a sequence $\left\{Y_{p}\right\}$ of independent random variables defined on the same probability space on which $X_{y}$, are defined and satisfying the following condition:

(8) the chatruteristic fauction of $X_{p}$ is

and

$$
P\left(X_{p}=0\right)=1 \text { if } p \in Q,
$$

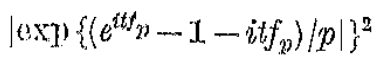

$$
P\left(X_{p} \neq Y_{p}\right)=0\left(1 / p^{2}\right)
$$

Now the theorem follows from Lemmas 1 and 6 .

Proof of theorem 2. Let $\left\{\eta_{y}\right\}$ be a sequence of independent random variables with

$$
\mathrm{P}\left(\eta_{p}=x\right)=\left(1-\frac{1}{p}\right) \sum_{\substack{t \\ f\left(p^{t}\right)=x}} \frac{1}{p^{l}} .
$$

It is chasy to see that ([5]) if $f$ has a distribution, then $\sum_{p} \eta_{p}$ converges almost overywhere and the distribution of $f$ coincides with $L\left(\sum_{n} \eta_{p}\right)$. Let $\left\{X_{p}, Z_{q}: p, q\right.$ primes $\}$ be a set of independent random variables defined on the same probalbility space on which $\left\{\eta_{p}\right\}$ are defined and satisfying the following conditions:

$$
\begin{gathered}
\mathrm{P}\left\{f(p) X_{p} \neq \eta_{p}\right\}=o\left(\frac{1}{p^{2}}\right), \\
\mathrm{P}\left\{Y_{p}=\ldots\right\}=\mathrm{P}\left\{Z_{p}=-k\right\}=\frac{1}{p^{k}}\left(1-\frac{1}{p}\right), \ldots k=0,1, \ldots
\end{gathered}
$$

Sinco $L\left(\sum \eta_{p}\right)$ is absolutely continuous, by temma 1 , it follows that $L\left(\sum_{p} f(p) \stackrel{p}{X_{p}}\right)$ is absolutely continuous. Consequently $L\left(\sum_{p} f(p)\left(Y_{p}+Z_{p}\right)\right)$ is absolutely continuons. Again by Tornma 1 and from the proof of Theorem 1. it follows that the distribution of $f(m)-f(m+1)$ is absolutely continuons. This complotes the proof of Theorem 2.

Oorollary 1. now follow easily from the above two theorems.

Proof of Gorollary 2. Since $f(m)$ is bounded, $\sum_{p} f(p)$ converges wholntely and hence $|f(p)|<1$ for all sufficicntly $\operatorname{large} p$. So for $N$ sufficiently liarge, wo have

$$
\sum_{p>N} \frac{f(p)^{2}}{p}\left(\sum_{p>N} f(p)^{4}\right)^{1 / 2}\left(\sum_{p \geqslant N} \frac{1}{p^{2}}\right)=O\left(\left(\sum_{p>N} \frac{1}{p^{1+1}}\right)^{1 / 2}\right) \cdot \frac{1}{N^{1 / 4}}=O\left(N^{-1 / 4}\right) .
$$


Now this corollary follows from Corollary 1.

Proof of Theorem 3. If a real additive arithmetic function $g$ satisfies (2), then by a result of Wixsing [7] there exists a constant $D$ and a bounded real additive arithmetic function $f$ such that

$$
g(m)=D \log m+f(m) \text { for } \quad m=1,2, \ldots
$$

Sinee $f$ is bounded, it satisfies condition (1). So the distribution of $f(m)-$ $-f(m+1)$ is singular. But $g(m)-g(m+1)=f(m)-f(m+1)+o(1)$ as $m \rightarrow \infty$. Tence the distribution of $g(m)-g(m+1)$ is the same as that of $f(m)-f(m+1)$. Consequently the distribution of $g(m)-g(m+1)$ is singular.

Proof of Theorem 4. Define a function $g(t)$ by

$$
\log g(t)=\sum_{p}\left(e^{i t t_{p}}-1-i t f_{p}\right) \frac{1}{p}
$$

Since $\left\{\exp \left(\frac{1}{p}\left(e^{i t f_{p}}-1-i t_{p}\right)\right)\right\}$ is the characteristic function of a contred Poisson random variable and sineo

$$
\sum_{p} \frac{f_{p}^{2}}{p}<\infty
$$

and $h_{h b}(t)=\exp \left\{\sum_{p \leqslant q}\left(e^{i t t_{p}}-1-i t f_{p 2}\right) \frac{1}{p}\right\}$ is a characteristic funotion for each $n, h_{n}(t)$ converges absolutoly and uniformly to $g(t)$ in every bounded interval, and $g(t)$ is an infinitely divisible characteristic function. As in the proof of Theorem 1 , it is sufficient to show that the distribution corresponding to $g(t)$ is absolutely contimuous.

Note that since

$$
|\sin y| \geqslant|y| / 2 \text { if } \quad|y| \leqslant \frac{1}{2},
$$

we have for any $\varepsilon>0$

i.e.

$$
\frac{1}{|\log 2 \varepsilon|} \sum_{p}^{\top} \frac{\left(\sin \left(f_{p} / 2 \varepsilon\right)\right)^{2}}{p} \geqslant \frac{1}{6 \varepsilon^{2}|\log 2 \varepsilon|}\left(\sum_{\left|f_{x \mid}\right|<s} \frac{f_{p}^{2}}{p}\right)
$$

We have

$$
\liminf _{\varepsilon \rightarrow 0} \frac{1}{|\log \varepsilon|} \sum_{p}\left\{\frac{\left(\sin \left(f_{p} \mid \varepsilon\right)\right)^{2}}{p}\right\}>\frac{1}{4}
$$

$$
\begin{aligned}
& \log |g(2 u)|^{2}=\left\{\sum_{p}\left(e^{i 2 u f_{p}}-1-i 2 u f_{p}\right) \frac{1}{p}\right\}+\left\{\sum_{p}\left(e^{-i 2 u f_{p}}-1+i 2 u f_{p}\right) \frac{1}{p}\right\} \\
= & \sum_{p}\left(e^{i 2 u f_{p}}+e^{-i 2 u f_{p}}-2\right) \frac{1}{p}=\sum_{p}\left(2 \cos 2 u f_{p}-2\right) \frac{1}{p}=-2 \sum_{p} \frac{2\left(\sin u f_{p}\right)^{2}}{p} .
\end{aligned}
$$

So,

Henes,

$$
-\frac{1}{2} \log |g(2 u)|=\sum_{p} \frac{\left(\sin u f_{p}\right)^{2}}{p}
$$

i.c.

$$
\liminf _{u \rightarrow \infty}\left\{-\frac{1}{2}(\log |g(2 u)|) \frac{1}{|\log u|}\right\}>\frac{1}{4}
$$

$$
\liminf _{u \rightarrow \infty}\left\{-(\log |g(u)|) \frac{1}{|\log u|}\right\}>\frac{1}{2}
$$

Hence for some $\delta>0$,

$$
|g(u)|=O\left(|u|^{-\frac{1}{2}-\delta}\right) \text { as } \quad u \rightarrow \infty
$$

So $g(u)$ is squire integrable, consequently, by Plancherel's theorem, it follows that the distribution function eorresponding to $g$ is absolutely contimuous. This completes the proof of Theorem 4.

The proof of Theorem 7 is similar to the proof of Theorem 4 .

Proof of Theorem. 5. Define

$$
f(p)= \begin{cases}a_{p} & \text { if } \quad p \phi Q, v(p) \neq 0 \text { and }|f(p)|<1 \\ 0 & \text { otherwise. }\end{cases}
$$

Let $v(F, p) \leqslant \%$ for all $p$.

In viow of Theorem 1 of [1] and Lemma 1, it follows that the distribution of $f(F(m))$, if it exists, is singular iff the distribution function corresponding to the characteristic function

is singular.

$$
h(t)=\exp \left\{\sum_{p}\left(e^{i t a_{p}}-1-i t a_{p}\right) \frac{r(F, p)}{p}\right\}
$$

Dofino

$$
s(t)=\operatorname{axp}\left\{\sum_{r(h, p)<h}\left(e^{\left.i l a_{p}-1-i t a_{p}\right)} \frac{(k-r(F, p))}{p}\right\}\right.
$$

Since $\frac{\sum}{n} a_{n}^{2} / p$ is linitie, $s(t)$ detinos a characteristic function and $|g(t)|^{2 k}$

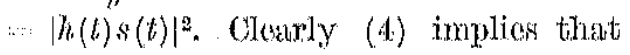

$$
\sum_{x \rightarrow N} a_{p}^{2} / p=0\left(\frac{1}{N^{i}}\right) \quad \text { as } \quad N \rightarrow \infty
$$

So tile distribution function corresponding to $|g(t)|^{2 / 2}$ is singular by Lemma 6 and hence tho distuibution corresponding to $h(t)$ is singular. This completies the proof of Theorem 5 . 
Proof of Theorem 6. It is not difficult to show from the proofs of Theorems 1,2 of $[1]$ and from lemma 1. that the distribution of $f(f(m))$ $-f(F(m+1))$ exists and is singular iff the distribution function corresponding to the characteristic function

$$
g^{*}(t)=\exp \left\{\sum_{p}\left(e^{i l a_{p}}+e^{-i k k_{p}}-2\right)\left(r^{*}(t, p) / p\right)\right\}
$$

is singulax, where $a_{p}$ is as atetined in Thoom 5 and $r^{*}\left(F^{7}, p\right)+r^{2}\left(h^{2}, p\right)$. $\left(r^{*}(T, p)<r(F, p)\right.$ for some $p$ if thoxe axist two foctors $P(m)$ and $Q(m)$ of $I(m)$ such that $Q(m)=P(m+1)$ for all $m$.

From Lemma 6 and from the proof of rheorom 5 it ansily thollows that the distribution corresponding to $g^{*}(t)$ is singulax. This completess the proof of Theorem 6 .

Remark. Let $F \in P$ and tet the degree of $F$ be $>1$. Sumpose titat condition (3) is satisfied and there exists a set $Q$ of prime numbers such that

(10) $\sum_{p \in Q} \frac{1}{p}<\infty$ anel $p \phi Q$ implies either $r(H, p) \neq 0$

$$
\text { or } f(p)=0 \text { and } r(h, p)=0
$$

Then we have the following

PRoposturon. If the distribution of $f(n)-f(n+1)$ exists and is ansolutely continuous, then the distribution of $f(F(m))-f(F(m+1))$ atso extsts and is absolutely continuous.

The proof of this proposition is similar to the proof of Proposition 3 of [2].

Note that condition (10) is satisfied if $F$ is divisible by a linear polynomial. Condition (10) cannot be replaced, since if $(10)$ is violated, then the following example shows that the distribution of $f(m)-f(m+1)$ is absolutely continuous but $f(F(m))-f(F(m+1))=0$ for all $m$.

Define a strongly additive arjithmetic tunction by

$$
f(p)=\left\{\begin{array}{l}
\frac{1}{(\log \log p)^{3 / 2}} \\
0 \quad \text { othorwise. }
\end{array} \text { if } p<\theta^{\circ} \text { and } p \text { anos } 3\right. \text { (m) }
$$

Take $F(m)=m^{2}+1$.

It is known ([2]) that if $p=3(\bmod 4)$ thon $p+m^{2}+1$ for any $m$ so $f(F(m))-f(F(m+1))=0$ for all $m$. But, on the other hand, it is not difficult to show that the distribution of $f(m)-f(m+1)$ is absolatioly continuous (see [2]).
Acknowledgements. The whithor expresses his indebtedness to Prof. J. K. Ghosh, whose suggestions and careful editing helped this article to assume itis present form. He also wishes to thank Prof. W. M. Paul for the rarious discussion he had with him.

\section{Theforences}

[1] G. I. Babu, On the distrilndion of additive avithmetical functions of integral poly-

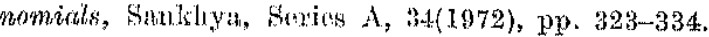

[2] - Some requlls on the distribution of oddilive arithmetio functions II, Aotia Arith. 23: (1973), pp. 315-328.

[5] T. Had dos, On the smoothness of the asymptotio distribution of additive arithmetical funotions, Amer. Journ. Matil. 61 (1939), pp. 722-725.

[4] - On the sum $\sum_{k=1}^{x} a(f(b))$, London Math. Soc. Journ. 27 (1952), pp. 7-15.

[5] J. Kubilius, lrobubilistics methods in the theory of numbers, Tranclations of Matih. Monographs, vol. Il, A.M.S., Providence 1964.

[6] M. Tan alsa, On the number of prime factors of integers, Japan J. Math. 25 (1955), pp. $I-20$.

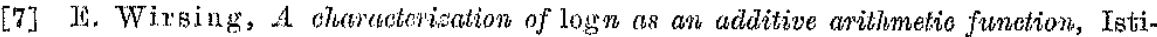
taxto Nazionala Di Altia Matillematica Symposia Mathornatica, Vol. IV, 1970, pp. $45-57$.

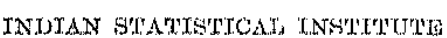

